\title{
Oncofertility: a grand collaboration between reproductive medicine and oncology
}

\author{
Teresa K Woodruff \\ The Thomas J Watkins Professor of Obstetrics and Gynecology, Department of Obstetrics and Gynecology, Feinberg \\ School of Medicine, Northwestern University, 303 E Superior Street, Lurie 10-250, Chicago, Illinois 60611, USA
}

Correspondence should be addressed to TKWoodruff; Email: tkw@northwestern.edu

\begin{abstract}
In 2007, I was asked by the University of Calgary to participate in a symposium called 'Pushing the Boundaries - Advances that Will Change the World in 20 Years'. My topic was oncofertility, a word I had just coined to describe the intersection of two disciplines oncology and fertility - and I was thrilled to share my passion for this new field and help young women with cancer protect their future reproductive health. Fertility preservation in the cancer setting lacked a concerted effort to bridge the disciplines in an organized manner. In early 2015, I was delighted to deliver a presentation for the Society for Reproduction and Fertility titled 'Sex in Three Cities', where I gave an update on the oncofertility movement, a remarkable cross-disciplinary, global collaboration created to address the fertility preservation needs of young cancer patients. During my tour of the UK, I was impressed by the interest among the society and its members to engage colleagues outside the discipline as well as the public in a dialogue about cutting-edge reproductive science. In this invited review, I will describe the work of the Oncofertility Consortium to provide fertility preservation options in the cancer setting and accelerate the acceptance of this critical topic on a global scale. I hope that one day this word and field it created will change the world for women who had been left out of the equation for far too long.

Reproduction (2015) 150 S1-S10
\end{abstract}

\section{Introduction}

Young cancer patients, facing the devastating news that their early careers, college plans, or childhood playtime will be forever changed by the fight against a ruthless disease, are often unaware that the life-preserving treatments they will undergo can also threaten their future fertility (Lobo 2005, Meirow et al. 2005, Jeruss \& Woodruff 2009, Barrett et al. 2010, De Vos et al. 2014, Wallace et al. 2014). Additionally, the potential loss of endocrine support of hormonally responsive tissues can trigger a cascade of long-term medical and quality-of-life problems. Beyond cancer, several non-malignant diseases and conditions, as well as their treatments, can also negatively affect reproductive function (HirshfeldCytron et al. 2011).

Ideally, the impact of a disease or its treatment on future fertility and endocrine health should be addressed as part of the initial comprehensive care plan for young patients, but the decision to protect fertility from the damaging effects of treatments, like radiation and chemotherapy, is complicated by the patients' age, marital status, whether they can delay treatment, and sometimes, the uncertainty of survival. The juxtaposition of a cancer diagnosis and the risk of future infertility in young adults and children can be overwhelming for individual patients and their parents and partners, and contemplating the impact of cancer treatment on fertility may be nearly impossible at a time when the focus is on survival. The conversation about reproductive health in young patients diagnosed with a fertility-threatening disease or treatment is particularly complex, covering topics from basic biology, medical practice planning, health access, and reproductive rights, to ethical, social, moral, cultural, religious, and personal perspectives. The conversation includes many stakeholders - practitioners, patients, parents, and partners, and even the public, policymakers, and advocates (Woodruff \& Snyder 2007, Dolin et al. 2010, Woodruff et al. 2010, 2013, Gracia \& Woodruff 2012).

A decade ago, fertility preservation in the cancer setting was not a new idea, but there was no coordinated effort to link cancer care and fertility management - even existing centers of excellence may have had only a single practitioner who managed care in an ad hoc manner. To better meet the fertility and endocrine health needs of young women and girls facing any fertility-threatening condition or treatment, the Oncofertility Consortium was created in 2005 (http://oncofertility.northwestern. edu/; Woodruff 2010; Table 1). The initiative began in North America, but there are many more programs developing around the globe (Ataman et al. 2015). 
Table 1 List of valuable oncofertility online resources with URLs.

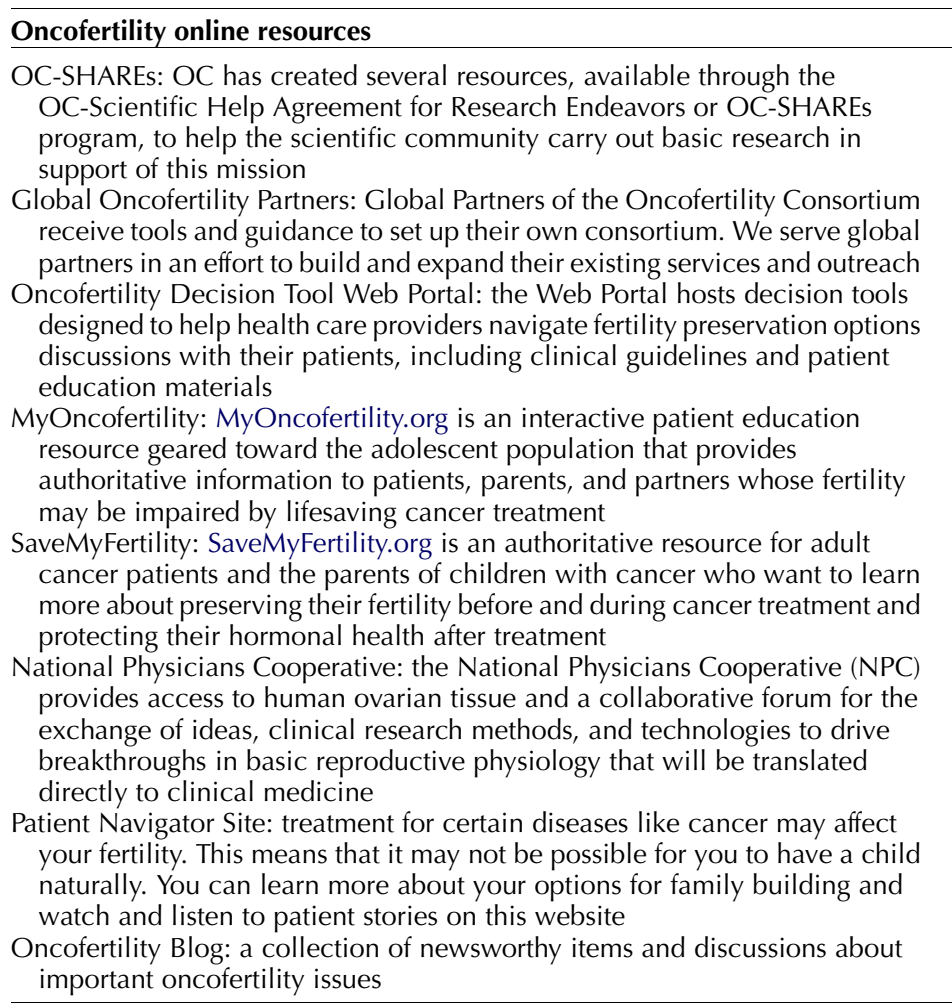

Website links
http://oncofertility.northwestern.edu/oncofertility-consortium-
scientific-help-agreement-research-endeavors-shares-program

http://oncofertility.northwestern.edu/global-oncofertility-partners

http://oncofertility.northwestern.edu/ODT-web-portal

http://www.myoncofertility.org/

http://www.savemyfertility.org/

http://oncofertility.northwestern.edu/physicians/ about-the-national-physicians-coop-npc

http://preservefertility.northwestern.edu/

http://blog.oncofertility.northwestern.edu/
Moreover, there are a number of guidelines that have been issued from societies around the globe that provide guidance to clinicians regarding fertility preservation and restoration (http://oncofertility.northwestern.edu/ ODT-web-portal\#guidelines). In this review, I would like to take you through the first decade of the Oncofertility Consortium - where we were when it started and where we stand today.

\section{Scope of the problem}

Advances in cancer diagnosis and the introduction of new cancer treatments have dramatically improved the odds of survival, permitting patients and practitioners to think well beyond the cure to future quality of life. Unfortunately, these life-saving treatments can also have a profoundly negative impact on reproductive endocrine function and fertility. In 2006, it was estimated that nearly 750000 young women in the USA had their childbearing years interrupted to undergo cancer treatment. Among these female cancer survivors who were under the age of 40 at diagnosis, the chance of achieving pregnancy was $20 \%$ lower in those diagnosed as children and $50 \%$ lower in those diagnosed as young adults compared to female siblings without cancer (Green et al. 2009a). When extrapolated globally, this level of infertility and its consequences on survivors' quality of life represent an enormous unmet need.
Although cancer is often thought of as a disease of aging, the number of young patients whose reproductive futures may be affected by cancer treatment is not small. In the USA in 2014, more than 1.6 million patients received a cancer diagnosis (National Cancer Institute \& Surveillance Epidemiology and End Results Program 2014a) and $\sim 14$ million were diagnosed worldwide (World Health Organisation 2015). In the USA, 23\% of new cancer diagnoses in 2007-2011 were made in patients younger than 45 years of age (National Cancer Institute \& Surveillance Epidemiology and End Results Program 2014a), a time when many may be contemplating or actively building their families. It is these patients for whom a reproductive consult should be considered at the time of diagnosis to address options for preserving fertility - before cancer treatment begins (Lobo 2005, Meirow et al. 2005, Jeruss \& Woodruff 2009, Woodruff 2010, De Vos et al. 2014, Wallace et al. 2014).

Children with cancer represent a special population of individuals for whom the cancer survival statistics have been most impressive - $\sim 85 \%$ of prepubertal cancer patients (age 0-14 years) are likely to survive their disease (National Cancer Institute \& Surveillance Epidemiology and End Results Program 2014b, Smith et al. 2014). For this reason, addressing the late effects associated with cancer treatment, such as cardiac dysfunction, neurocognitive impairment, and reproductive and endocrine issues, has taken on a new urgency. Cancer treatments may also cause many young patients 
to experience difficulty transitioning through puberty, with loss of bone and muscle development and delayed development of secondary sex characteristics. Understanding the potential effects of cancer treatment on both short- and long-term reproductive and endocrine health, and how to mitigate these risks before treatment begins, presented a daunting challenge.

\section{Minding the gaps}

Back in 2005, when oncofertility programs were just starting, men and pubertal boys were regularly offered sperm banking as an option to preserve their fertility prior to cancer treatment. Yet young women who had the same hope for survival had few to no fertility preservation options made available to them. There were three main gaps that created this chasm in care - an information gap, a data gap, and an option gap (Woodruff 2010). With regard to the information gap, there was limited dialogue between oncology and fertility specialists about the fertility concerns of young cancer patients (Kohler et al. 2011). There was also a general reluctance among oncologists to discuss reproductive issues with young patients and their parents (Quinn et al. 2012). They would talk about how patients might lose their hair follicles because of their cancer treatment but were not comfortable talking about how patients might lose their ovarian follicles.

A cancer diagnosis in a young person seems particularly cruel and is understandably accompanied by an urgency to begin treatment. As such, many practitioners may assume that parents are solely interested in the survival of their child, not the some far-off future risk to fertility or endocrine health. Yet our early assessment of the attitudes of adult survivors of childhood cancer told a very different story - young women and their parents alike wished that they had fertility preservation options made available to them before they started treatment (Nieman et al. 2006, 2007, Gorman et al. 2012). For many of these adult survivors, their cancer treatment had become a distant memory, but the issue of infertility remained as a real and present problem that limited their social life and curtailed their hopes of a biologic family.

The data gap further hindered the discussion - both between oncologists and fertility specialists and between oncologists, patients, and parents - since little was known about the fertility effects of specific cancer treatments. It was, and continues to be, very difficult to be precise about the risks posed by specific cancer treatments on fertility and reproductive function. Several studies have demonstrated that cancer therapies destroy ovarian follicles and accelerate ovarian aging, influence puberty and menstruation, and shift the age of menopause earlier (Goodwin et al. 1999, Sklar et al. 2006, Gracia et al. 2012, Johnson et al. 2013). One study estimated that cancer treatment accelerated ovarian aging by 7 years, meaning that the ovaries of a 30-yearold cancer survivor would function like the ovaries of a 37-year-old (Levine et al. 2015). The best available evidence on the effect of cancer treatment comes from the Childhood Cancer Survivor Study (CCSS), a longitudinal cohort study of cancer survivors who were younger than 21 years of age at diagnosis compared to a sibling control group. In one analysis, female cancer survivors were more likely to have clinical infertility ( $>1$ year of attempts to conceive) and the risk increased with exposure to increasing doses of radiation and chemotherapy with alkylating agents (Green et al. 2009a, 2011, Barton et al. 2013). Cancer survivors exposed to pelvic radiation or high-dose alkylating agents or procarbazine were also more likely to experience acute ovarian failure and premature menopause (Green et al. 2009b). Exposure to more than 7.5 Gy to the testes or treatment with high doses of alkylating agents, cyclophosphamide, or procarbazine was associated with lower fertility among men enrolled in the study (Green et al. 2010). The lack of information about the specific effects of certain cancer treatments and regimens on various markers of reproductive and endocrine function continues to be a major issue for the field, as long-term studies such as the CCSS are costly and difficult to manage.

The final issue was the option gap. Even though emergency IVF - performed in the window between cancer diagnosis and treatment - was available in 2005, it was rare that this option would be offered to young women with a newly diagnosed cancer, and there was no easy way to quickly navigate patients from oncology to fertility care and back again. Reproductive endocrinologists and fertility specialists were not accustomed to handling rapid consults for patients who required urgent care prior to undergoing cancer treatment. Moreover, because this intervention was not appropriate for all patients, oncologists were reluctant to engage in a dialogue about it, knowing some patients with aggressive disease or hormone-responsive disease would have no ability to alter the course of their treatment to undergo hormonal stimulation for IVF.

As we worked through these gaps, it also became clear that fertility concerns touch more than just those patients affected by cancer. For example, gastrointestinal diseases, rheumatologic disorders, non-malignant hematologic conditions (most prominently $\beta$-thalassemia), neurologic disorders, renal disorders, gynecologic conditions, and metabolic diseases can adversely impact the reproductive axis, yet there is little information about these effects or what fertility preservation options may be provided (Hirshfeld-Cytron et al. 2011). Moreover, the negative iatrogenic effects of various treatments and procedures for non-malignant diseases on reproductive function are only now being appreciated. Just as for cancer patients, these diseases and treatments can lead to impaired gonadal function, endocrine function, 
sexual function, or ability to carry a pregnancy to term. We know that cancer patients are interested in fertility interventions, but clinical practice is only now recognizing the need for education and advocacy for this broader group of patients. In response, our focus expanded to ensure that all patients facing a disease or treatment that limits reproductive function can benefit from the findings of basic and clinical reproductive research in fertility preservation and that breakthroughs made and technologies developed at the bench are translated to clinical practice rapidly and effectively.

\section{The Oncofertility Consortium}

When it was first established, the Oncofertility Consortium worked to address the first and last gaps - the information gap and the option gap - by educating providers and allied health professionals across disciplines about fertility preservation in the cancer setting. We facilitated cross-disciplinary cooperation between oncologists and reproductive endocrinologists, engaged the public and broadened the discussion about oncofertility, and accelerated the pace and quality of basic and clinical research to develop a larger range of fertility preservation options, including biological and nonbiological options for males and females, pubertal and young adult patients (Woodruff 2010, Waimey et al. 2013). Some of these options remain experimental, while others are now standard of care for fertility preservation in the cancer setting.

The mission of the Oncofertility Consortium was to serve as an authoritative voice for cancer patients facing fertility-threatening treatments and to create new corridors of discovery and dialogue between research and clinical practice at the intersection of oncology, pediatrics, reproductive science, policy research, reproductive health law, bioethics, communication science, and cognitive and learning science (Woodruff \& Snyder 2007, Woodruff 2010). On the scientific side, we wanted to address fundamental questions of ovarian follicle growth and maturation as well as discover how follicles could be preserved without appreciable damage for long-term storage. For children with cancer who cannot undergo emergency IVF to retrieve mature oocytes or produce embryos for cryopreservation, retrieving ovarian tissue and preserving the immature follicles is their only remaining option to preserve fertility. Figuring out how to cryopreserve these small follicles and then recover them at a later date to restore fertility and endocrine function required the scientific community to come together in new and innovative ways.

We also had an existing unmet clinical need. Approximately 130000 women receive a cancer diagnosis in the USA each year (National Cancer Institute \& Surveillance Epidemiology and End Results Program 2014a), and some sort of fertility consultation pathway that addressed the fertility threat, or lack of a threat, needed to be implemented. In cases in which intervention was also required, we needed plans for Institutional Review Board (IRB)-approved experimental options and new lines of communication between oncology and reproductive endocrinology. We needed legal, ethical, and insurance and reimbursement issues to be addressed (Campo-Engelstein 2010a,b, 2011, Dolin et al. 2010). These were complicated and interconnected issues, but we recognized that any discovery we made at the bench would not be translated into clinical care on the rapid time scale required or with the broad quality metrics across practices that would be in the best interest of patients. Finally, we needed to broadly educate pediatric and adult oncologists and reproductive endocrinologists and urologists to understand the nature of the fertility concerns of cancer patients and their parents and integrate a new patient category with unique care requirements into an already busy program (Jeruss \& Woodruff 2009, Gardino et al. 2010, Redig et al. 2011, Gracia \& Woodruff 2012). These complex decisions required the assembly of large teams, which could be unwieldy, but once assembled and functional, they could shift the burden from an individual to a broad team to achieve our goals.

Since its founding, the Oncofertility Consortium has pursued five solution-guided objectives (Waimey et al. 2013, Woodruff 2013). We developed and applied new biomaterials for the ex vivo and in vivo growth of ovarian follicles with the ultimate goal of providing new options for preserving both fertility and endocrine function in patients. We also designed and tested mitigation strategies to reduce the off-target effects of chemotherapy on oocyte function and thereby protect future endocrine function - we hope that 1 day these strategies will eliminate the need for oncofertility. We developed and tested physician- and patient-guided tools to increase the inclusion and adherence of patients at risk of negative iatrogenic reproductive effects in fertility preservation studies. We created and disseminated patient-, provider-, and public-facing didactics and, finally, formally supported the training of the next generation of leaders at the intersection of reproductive health and disease care. Even as we created our USA-based consortium of physicians and allied health professionals, basic and clinical researchers, patients, and advocates, we also reached out globally to increase the awareness of oncofertility and fertility preservation and to catalyze research and its translation to patient care through a shared vision, shared technology and resources, and a shared commitment to patients and the public. We understood that outstanding science and patient-facing activities happening around the world could be better harnessed if we all worked together and shared best practices to create comprehensive care plans. As a result, there are now centers in 19 countries actively engaged in the global oncofertility community. 


\section{Fertility preservation decision-making}

Making decisions about preserving future endocrine function and fertility requires that patients weigh information from many different sources - their doctors, their families, society - at a highly emotional time, when they are facing an existential crisis about themselves, their survival, and their future (Clayman et al. 2007, Gardino et al. 2010, Woodruff et al. 2010, 2013, Gracia \& Woodruff 2012, Murphy et al. 2012, Quinn et al. 2012, Quinn \& Vadaparampil 2013, Duncan et al. 2014). As with any decision, patients need to be fully aware of and understand their options, but this can be particularly challenging for patients who have just received a cancer diagnosis and must make a decision within a tight time frame. Fertility interventions in the conventional infertility setting are not urgent and patients are often well aware of their fertility status and are some of the most well-educated consumers of health information. In contrast, the young cancer patient is not generally aware of their reproductive health or fertility concerns, so the discussion about fertility options requires a new kind of language to describe the potential impact of their treatment on endocrine and reproductive outcomes. Finally, there are issues of geographic access to care and financial constraints that feed into the decision-making process.

One of the ways we have found to significantly facilitate fertility preservation decision-making is through the use of a patient navigator (http://oncofertility.northwestern.edu/patient-navigators). The oncofertility patient navigator at Northwestern University is well-versed in the patient's oncology diagnosis and treatment plan, as well as the fertility consequences of the planned treatment and the available fertility preservation options. The navigator can further the discussion and readily move the patient from oncology to reproductive endocrinology services and then back to cancer care as quickly as possible (Scott-Trainer 2010, Gracia \& Woodruff 2012). The Oncofertility Consortium has also made available a decision tool web portal, where health care providers can find various decision tools and resources to help them work through the available fertility preservation options with their patients (http://oncofertility.northwestern.edu/ODTweb-portal\#DecisionTools).

Another patient-targeted approach was the development of a network of providers so that patients are able to receive care closer to home (the Oncofertility Consortium National Physicians Cooperative (NPC), http://oncofertility.northwestern.edu/physicians/aboutthe-national-physicians-coop-npc; the Global Oncofertility Network, http://oncofertility.northwestern.edu/ global-oncofertility-partners; and a growing Pediatric Oncofertility Network, http://oncofertility.northwestern. edu/pediatric-oncologists). This represented a radical shift, from only a few centers of excellence to more evenly distributed and standardized oncofertility care available across the country, and now globally. The NPC has grown to 59 clinical sites nationwide, including 20 pediatric clinical sites. The NPC shares its collective practice experience by providing the NPC users manual, IRB templates, and billing resources to practices considering the implementation of their own fertility preservation programs (Gracia \& Woodruff 2012). The NPC also obtains donated human ovarian tissues and oocytes under IRB-approved protocols for basic investigation and distributes them across the network for research purposes. The activities of the NPC member sites have resulted in a substantial collection of rare research tissue, with more than 130 samples taken over the last 5 years, and the amount of research tissues collected has increased significantly in recent years.

Other tools to facilitate decision-making include a global fertility hotline with an oncofertility patient navigator available to physicians and patients (http:// oncofertility.northwestern.edu/fertline; 866-708-FERT); a patient-facing website called Myoncofertility.org that provides information to patients, parents, and partners about fertility issues and what can be done to preserve fertility at various points from diagnosis to treatment; a SaveMyFertility module (including a microsite, fact sheets, pocket guides, and mobile app) for providers; and a list of 'cancer friendly' adoption agencies. All of these patient educational tools are free and available for download and can be customized. The Oncofertility Consortium name and associated awareness ribbon are used together with the home institution's name to highlight the concerted global effort that is in place to address patient issues. Our goal is to provide authoritative, up-to-date, enduring educational materials that can be accessed anywhere, at any time, and in any language.

One of the most important things that has happened since the formation of the Oncofertility Consortium is the development of several society-based guidelines (Backhus et al. 2007, Ethics Committee of American Society for Reproductive Medicine (ASRM) 2013, Loren et al. 2013). The American Society of Clinical Oncology (ASCO), ASRM, and American Academy of Pediatrics (AAP) guidelines recommend the discussion of potential fertility impairment at the earliest possible moment after diagnosis, a prompt referral to a qualified specialist if the patient is interested, and the promotion of clinical trials to advance the state of knowledge in fertility preservation. ASRM, AAP, and European Society of Human Reproduction and Embryology (ESHRE) jointly stated: 'Parents may act to preserve fertility of cancer patients who are minors if the child assents and the intervention is likely to provide net benefits to the child' (Ethics Committee of American Society for Reproductive Medicine (ASRM) 2013). 


\section{Current options for fertility preservation}

The current options for men and pubertal boys include sperm banking, and if it is not possible to retrieve a semen sample, testicular sperm extraction, aspiration, or biopsy to obtain testicular tissue for cryopreservation is performed. Prepubertal boys can be consented to a protocol for a testicular biopsy. Work in the field of in vitro male germ cell development using biopsy tissues is ongoing in a number of centers (Orwig \& Schlatt 2005, Schlatt et al. 2009, Reuter et al. 2014, Valli et al. 2014). For women 16 years of age or older, the standard of care was to undergo hormone stimulation to obtain mature eggs for fertilization and cryopreservation of embryos prior to starting cancer treatment. Thanks to advances made in oocyte cryopreservation, today, these young women have the additional option of cryopreserving mature oocytes rather than trying to find a sperm donor for fertilization.

Neither of these options is available for women with hormone-sensitive cancers treated with estrogen antagonists including letrozole or for prepubertal girls who are unable to undergo hormone stimulation. For these patients, ovarian tissue cryopreservation is an experimental option, though it is currently reserved for those who have a very high likelihood of losing fertility. The goal is to be able to preserve the immature follicles within the ovarian tissue so that they can be used at a later date to restore reproductive function and fertility. Retransplantation of ovarian tissue that has been cryopreserved through slow freeze protocols or vitrification has led to live births in humans (Dittrich et al. 2015). There remains uncertainty regarding the efficiency of the method, because the total number of transplants that have been performed is unknown, and there is a very real risk that the transplanted tissue can reintroduce cancer cells into the recipient cancer survivor. Ovarian tissue transplantation has also been used to transition prepubertal patients through puberty (Poirot et al. 2012, Anderson et al. 2013). The fact that surgeons are able to use stored ovarian tissue to replace gonadal function is important and suggests that the tissue that is currently being stored will likely be of future use by patients; however, it is critical that we find ways to reduce the risk of disease transmission.

\section{Advances in ovarian follicle culture}

To offer hope of fertility to a greater number of girls and young women with cancer and other diseases in which sterility is the consequence of treatment, we must overcome the challenges of follicle growth and development in vitro. The Oncofertility Consortium has supported fundamental basic research on human ovarian follicle development and fertility, relying on the donations of ovarian tissue for research made to the NPC. This work has led to breakthroughs in our understanding of follicle biology and the effects of cancer treatments (Xu et al. 2011a), as well as new techniques in cryopreservation (Backhus et al. 2007, De Vos et al. 2014) and in vitro follicle growth and maturation (Laronda et al. 2014) that may offer greater options for fertility preservation to the youngest of cancer patients.

The ovarian follicle is a unique structure that contains the oocyte surrounded by and intimately connected to support cells. The normal architecture of the human follicle cannot be maintained in traditional two-dimensional tissue culture dishes. In collaboration with Lonnie Shea, a biomedical engineer, our investigative group developed a three-dimensional culture system that supports follicle development, largely, we believe, by maintaining the links between the gamete and its surrounding cells (Kreeger et al. 2003, Pangas et al. 2003). Using this system, we have shown that the follicle is a fully autonomous unit, able to dictate its activities with very little support from the external environment. When we started our work, we expected that a great deal of manipulation would be required to get the system going. It turns out that the more we did, the less happy our follicles were. We have always known that follicles require follicle-stimulating hormone to stimulate granulosa cell proliferation and hormone production and this turned out to be true in the in vitro setting as well (Kreeger et al. 2005). We also found that the best quality eggs came from oocytes that had been matured in the context of the somatic cells throughout the entire growth phase (Tingen et al. 2011, Tagler et al. 2012, Hornick et al. 2013). Further, the role of the alginate in the follicle culture system is quite simple: to provide a physical support system that maintains the follicle architecture and interaction between the oocyte and the surrounding granulosa and theca cells. What was unclear was whether the physical environment played an active role in the function of the follicle. When we first engineered our follicle culture system, we happened to use very weak gels - serendipitously, we ended up discovering that the more rigid the gel, the less growth of the follicle and the poorer the quality of the resulting egg (West et al. 2007, Woodruff \& Shea 2011). So hormones, structure, and environment were the three keys to ovarian follicle maturation, and our current focus is on understanding how to get all three of these contributing factors integrated at the right time and at the right level to produce healthy oocytes in vitro.

Ultimately, it is our hope that in vitro follicle culture technology will be applied clinically to grow immature follicles recovered from cryopreserved ovarian tissue to produce mature oocytes for use in IVF, allowing pediatric patients to 1 day utilize the immature follicles in their stored ovarian tissues to restore future fertility. Thus far, we have grown secondary mouse follicles in alginate that can be matured and fertilized in vitro to produce live offspring (Xu et al. 2006), and we have been able to support primordial, primary, and secondary follicle development from cryopreserved tissue from various animal models (Xu et al. 2009a), nonhuman primates 
(Jin et al. 2010, Hornick et al. 2012, Xu et al. 2013a) and humans (Xu et al. 2009b, Barrett et al. 2010, Laronda et al. 2014). We have also been able to recapitulate the process of ovulation and form a luteal body with cultured mouse follicles, achieving a full 'menstrual cycle in a dish' (Skory et al. 2015). We have matured eggs from human follicles (Xiao and T Woodruff unpublished observations) but have not fertilized or activated these eggs because of legal restrictions regarding the use of human gametes for research in the USA (Tingen et al. 2010, Campo-Engelstein et al. 2011, Rodriguez et al. 2011).

Other groups around the globe are also developing three-dimensional follicle culture technologies, and there are similar advances being made in the goat, dog, and baboon (Songsasen et al. 2011, Xu et al. 2011b, Silva et al. 2014). In the monkey, Richard Stouffer et al. have achieved in vitro oocyte maturation, fertilization, and early embryonic development (Xu et al. 2009c, 2010, 2011c, 2013b, Fisher et al. 2013, Rodrigues et al. 2015). The work is progressing, and many labs are continuing to unlock the mechanisms of follicle development, but we do not have a complete system that is ready for use with the tissue stored by young cancer patients. Importantly, advances being made in in vitro follicle growth are not the work of one lab but the concerted effort within the oncofertility field to ensure that the tissue that patients have consented for research protocols is being used to discover and develop new fertility preservation options that will 1 day help other young cancer patients. Patients give their consent with the awareness that we do not yet have the answers needed to provide assurance that their ovarian tissue can be useful outside of the transplant arena, but this is our goal.

Ultimately, it is my hope that 1 day we will not need to rely on preserving ovarian follicles to restore fertility and reproductive function after cancer treatment. As new, smarter therapeutics are created that can treat the disease without causing collateral damage to the ovary and testes, we will no longer have a need for oncofertility. We are learning more and more about the way the female egg and the male sperm respond to toxic drugs. Additionally, new technologies to more precisely target drugs and radiation, as well as biologics that are specific to the disease, are on the horizon. By coupling these technological advancements with a concerted effort to identify the triggers of cancer and refocus our efforts on preventive measures that will either reduce the incidence of disease or diagnose it earlier, we will achieve better outcomes for patients. All of these advances - in cancer prevention, diagnosis, and treatment - will 1 day make it possible to address cancer without affecting future reproductive health, making the word oncofertility and its practice obsolete.

\section{Expanding reproductive education}

One of the issues I have been most passionate about is the general understanding of reproductive health terms by the public. The general public and patients have a low comprehension of reproductive science terminology, which creates barriers to discussions about reproductive biology and health and makes health care decisionmaking difficult. To address this need, I created Repropedia, a website that is curated by members of the global reproductive science community and serves as an authoritative source of definitions for reproductive health terms (http://www.repropedia.org; Smeyers et al. 2012). Importantly, this site can be used to directly interact with any other website by providing pop-up definition boxes. This technology ensures that readers get information in context and do not have to leave their website of interest. This is important for the Oncofertility Consortium's public-facing blogs and news feeds (https:// twitter.com/oncofertility; http://oncofertility.northwestern.edu/news; and https://www.facebook.com/pages/ Oncofertility-Consortium/274654090671), ensuring that the definitions of reproductive terms are not stumbling blocks. Repropedia can be linked to any website or blog and, in so doing, improves the comprehension of reproductive terms found anywhere online. In the future, it is my hope that all people will develop a high reproductive science IQ with which they can make fully informed health decisions in a way that improves their individual health and the health of their offspring. The Oncofertility Consortium also focused on education of the next generation of reproductive scientists, clinical researchers, and reproductive health advocates and consumers by engaging high school girls as participants in the Oncofertility Saturday Academy (Faurot \& Woodruff 2010). The goal is to use oncofertility as a tool to interest students in the world of research in a fresh new way (http://oncofertility.northwestern.edu/ oncofertility-saturday-academies). These programs are provided across the USA (Northwestern University, Chicago, IL; University of California, San Diego; Oregon National Primate Research Center; and University of Pennsylvania), and the girls present their work at the annual Oncofertility Consortium meetings.

\section{Final thoughts}

Oncofertility was just an idea 10 years ago. Today it is a distinct field of medicine, offering new hope to cancer patients who will survive their disease, with options for preserving their fertility that prior generations lacked. In the last year alone, $80 \%$ of the young cancer patients at my institution received information about fertility as part of their cancer care. I cannot describe just how monumental this shift is for medical practice in North America and perhaps even globally, with reproductive specialists and oncologists working together to improve patient care. For the patients at my institution, the discussions taking place between these two very different disciplines have led to interventions - banking eggs, sperm, embryos, oocytes, or tissue - and 
discussions about contraception, with the goal of preserving the patient's option to have a future family.

From the bench to the bedside to babies, oncofertility is now part of the normal lexicon of centers of excellence around the globe, where oncologists and reproductive specialists make fertility after cancer a priority at the time of diagnosis (Woodruff 2013). Shared decision aids and tools for work such as IRB forms provide an altruistic environment in which busy oncologists and reproductive endocrinologists can stay up to date with medical recommendations and have access to the tools they need to implement a program at their institution. The successes in patient care have been paralleled by the efforts of reproductive scientists who are making discoveries in gamete biology that can be rapidly translated to the clinic and by social scientists who are delving into the complex social, ethical, and personal aspects of oncofertility to facilitate decision-making by patients and their caregivers. It is remarkable to think that less than a decade ago, I presented this kind of multidisciplinary, collaborative effort to improve patient care as the future of medicine. For many of our cancer survivors, that future is now, and they are the proud parents of children they thought they might not be able to have. My hope for the future is that we will ultimately eliminate the need for this field through more specific tools that treat the cancer and reduce unwanted side effects of the drugs. Until that time, we will continue to develop the field in a collaborative way that increases the pace of research and its translation into care.

\section{Declaration of interest}

The author declares that there is no conflict of interest that could be perceived as prejudicing the impartiality of the review.

\section{Funding}

The author receives funding through the following grants: the Center for Reproductive Health after Disease (P50HD076188) from the National Institutes of Health National Center for Translational Research in Reproduction and Infertility (NCTRI), and the National Institutes of Health Roadmap for Medical Research (5UL1DE019587).

\section{Acknowledgements}

The author gratefully acknowledges the leaders and members of the Oncofertility Consortium around the world. Specific thanks are due to Kristin Smith who serves as the global oncofertility patient navigator and is tireless in her efforts to provide sound advice to patients, providers, and this author regarding fertility interventions. The author also thanks Brigid Smith and Lauren Ataman who are the current administrative leaders of the Oncofertility Consortium. They are great partners in this work. The author also acknowledges Stacey Tobin, my former student and friend, who provides excellent editorial assistance to me, including for this editorial. I also wish to thank the Society for
Reproduction and Fertility and the opportunity to serve as this year's 'Sex in Three Cities' lecturer. It was a real treat meeting so many colleagues and friends across the UK and to interact with the next generation of leaders - your graduate students and fellows. The future of reproductive science and medicine is bright. The concept of a public-facing lecture in reproductive science is an important one and I was thrilled to meet interested laypeople and hope that their enthusiasm translates into support for your work. Finally, I want to acknowledge all of the colleagues who are making a difference in the lives of men and women with a cancer diagnosis. This editorial is necessarily myopic - it would take books to tell the story adequately. Therefore, the citations listed and the statement of progress above represents a tiny fraction of what has been done by leaders around the globe. I apologize for my inadequacy in this regard but am pleased to share a bit of the work done by the Oncofertility Consortium during its formative years.

\section{References}

Anderson RA, Hindmarsh PC \& Wallace WH 2013 Induction of puberty by autograft of cryopreserved ovarian tissue in a patient previously treated for Ewing sarcoma. European Journal of Cancer 49 2960-2961. (doi:10.1016/j.ejca.2013.04.031)

Ataman LM, Rodrigues JK, Marinho RM, Caetano JPJ, Chenin MB, Alves da Motta EL, Serafini P, Suzuki N, Furui T, Takae S et al. 2015 Creating a global community of practice for oncofertility. Journal of Global Oncology. In Press. (doi:10.1007/978-0-387-72293-1)

Backhus LE, Kondapalli LA, Chang RJ, Coutifaris C, Kazer R \& Woodruff TK 2007 Oncofertility consortium consensus statement: guidelines for ovarian tissue cryopreservation. Cancer Treatment and Research 138 235-239. (doi:10.1095/biolreprod.109.082933)

Barrett SL, Shea LD \& Woodruff TK 2010 Noninvasive index of cryorecovery and growth potential for human follicles in vitro. Biology of Reproduction 82 1180-1189. (doi:10.1095/biolreprod.109.082933)

Barton SE, Najita JS, Ginsburg ES, Leisenring WM, Stovall M, Weathers RE, Sklar CA, Robison LL \& Diller L 2013 Infertility, infertility treatment, and achievement of pregnancy in female survivors of childhood cancer: a report from the Childhood Cancer Survivor Study cohort. Lancet. Oncology 14 873-881. (doi:10.1016/S1470-2045(13)70251-1)

Campo-Engelstein L 2010a Consistency in insurance coverage for iatrogenic conditions resulting from cancer treatment including fertility preservation. Journal of Clinical Oncology 28 1284-1286. (doi:10.1200/ JCO.2009.25.6883)

Campo-Engelstein L 2010b For the sake of consistency and fairness: why insurance companies should cover fertility preservation treatment for iatrogenic infertility. Cancer Treatment and Research 156 381-388. (doi:10.1136/jme.2010.038588)

Campo-Engelstein L 2011 Gametes or organs? How should we legally classify ovaries used for transplantation in the USA? Journal of Medical Ethics 37 166-170. (doi:10.1136/jme.2010.038588)

Campo-Engelstein L, Rodriguez S, Tingen C \& Woodruff T 2011 Practical parthenote policy and the practice of science. American Journal of Bioethics 11 W1-W2. (doi:10.1080/15265161.2011.563162)

Clayman ML, Galvin KM \& Arntson P 2007 Shared decision making: fertility and pediatric cancers. Cancer Treatment and Research 138 149-160. (doi:10.1016/S0140-6736(14)60834-5)

De Vos M, Smitz J \& Woodruff TK 2014 Fertility preservation in women with cancer. Lancet 384 1302-1310. (doi:10.1016/S0140-6736(14)60834-5)

Dittrich R, Hackl J, Lotz L, Hoffmann I \& Beckmann MW 2015 Pregnancies and live births after 20 transplantations of cryopreserved ovarian tissue in a single center. Fertility and Sterility 103 462-468. (doi:10.1016/j. fertnstert.2014.10.045)

Dolin G, Roberts DE, Rodriguez LM \& Woodruff TK 2010 Medical hope, legal pitfalls: potential legal issues in the emerging field of oncofertility. Cancer Treatment and Research 156 111-134. (doi:10.1016/B9781-4557-2758-2.02001-1) 
Duncan FE, Brannigan RE \& Woodruff TK 2014 Fertility preservation. In Yen and Jaffe's Reproductive Endocrinology. $7^{\text {th }}$ Edition, Edited by JF Strauss III and RL Barbieri. Elsevier Canada.

Ethics Committee of American Society for Reproductive Medicine (ASRM) 2013 Fertility preservation and reproduction in patients facing gonadotoxic therapies: a committee opinion. Fertility and Sterility $\mathbf{1 0 0}$ 1224-1231. (doi:10.1016/j.fertnstert.2013.08.041)

Faurot M \& Woodruff TK 2010 The Oncofertility Saturday Academy: a paradigm to expand the educational opportunities and ambitions of high school girls. Cancer Treatment and Research 156 321-344. (doi:10.1007/978-1-4419-6518-9_25)

Fisher TE, Molskness TA, Villeda A, Zelinski MB, Stouffer RL \& Xu J 2013 Vascular endothelial growth factor and angiopoietin production by primate follicles during culture is a function of growth rate, gonadotrophin exposure and oxygen milieu. Human Reproduction 28 3263-3270. (doi:10.1093/humrep/det337)

Gardino SL, Jeruss JS \& Woodruff TK 2010 Using decision trees to enhance interdisciplinary team work: the case of oncofertility. Journal of Assisted Reproduction and Genetics 27 227-231. (doi:10.1007/s10815-0109413-8)

Goodwin PJ, Ennis M, Pritchard KI, Trudeau M \& Hood N 1999 Risk of menopause during the first year after breast cancer diagnosis. Journal of Clinical Oncology 17 2365-2370. (doi:10.1093/humrep/det337)

Gorman JR, Bailey S, Pierce JP \& Su HI 2012 How do you feel about fertility and parenthood? The voices of young female cancer survivors Journal of Cancer Survivorship 6 200-209. (doi:10.1007/s11764-011-0211-9)

Gracia C \& Woodruff TK 2012 In Oncofertility Medical Practice: Clinical Issues and Implementation. New York, NY: Springer Science+Business Media.

Gracia CR, Sammel MD, Freeman E, Prewitt M, Carlson C, Ray A, Vance A \& Ginsberg JP 2012 Impact of cancer therapies on ovarian reserve. Fertility and Sterility 97 134-140.e131. (doi:10.1016/j.fertnstert.2011.10.040)

Green DM, Kawashima T, Stovall M, Leisenring W, Sklar CA, Mertens AC, Donaldson SS, Byrne J \& Robison LL 2009a Fertility of female survivors of childhood cancer: a report from the Childhood Cancer Survivor Study. Journal of Clinical Oncology 27 2677-2685. (doi:10.1200/JCO.2008. 20.1541)

Green DM, Sklar CA, Boice JD Jr, Mulvihill JJ, Whitton JA, Stovall M \& Yasui Y $2009 \mathrm{~b}$ Ovarian failure and reproductive outcomes after childhood cancer treatment: results from the Childhood Cancer Survivor Study. Journal of Clinical Oncology 27 2374-2381. (doi:10.1200/JCO.2008.21.1839)

Green DM, Kawashima T, Stovall M, Leisenring W, Sklar CA, Mertens AC, Donaldson SS, Byrne J \& Robison LL 2010 Fertility of male survivors of childhood cancer: a report from the Childhood Cancer Survivor Study. Journal of Clinical Oncology 28 332-339. (doi:10.1200/JCO.2009.24.9037)

Green DM, Nolan VG, Kawashima T, Stovall M, Donaldson SS, Srivastava D, Leisenring W, Robison LL \& Sklar CA 2011 Decreased fertility among female childhood cancer survivors who received 2227 Gy hypothalamic/pituitary irradiation: a report from the Childhood Cancer Survivor Study. Fertility and Sterility 95 1922-1927 (1927.e1). (doi:10.1016/j.fertnstert.2011.02.002)

Hirshfeld-Cytron JE, Duncan FE, Xu M, Jozefik JK, Shea LD \& Woodruff TK 2011 Animal age, weight and estrus cycle stage impact the quality of in vitro grown follicles. Human Reproduction 26 2473-2485. (doi:10. 1093/humrep/der183)

Hornick JE, Duncan FE, Shea LD \& Woodruff TK 2012 Isolated primate primordial follicles require a rigid physical environment to survive and grow in vitro. Human Reproduction 27 1801-1810. (doi:10.1093/humrep/der468)

Hornick JE, Duncan FE, Shea LD \& Woodruff TK 2013 Multiple follicle culture supports primary follicle growth through paracrine-acting signals. Reproduction 145 19-32. (doi:10.1530/REP-12-0233)

Jeruss JS \& Woodruff TK 2009 Preservation of fertility in patients with cancer. New England Journal of Medicine 360 902-911. (doi:10.1056/ NEJMra0801454)

Jin S, Lei L, Shea LD, Zelinski MB, Stouffer RL \& Woodruff TK 2010 Markers of growth and development in primate primordial follicles are preserved after slow cryopreservation. Fertility and Sterility 93 2627-2632. (doi:10. 1016/j.fertnstert.2009.11.029)

Johnson LN, Dillon KE, Sammel MD, Efymow BL, Mainigi MA, Dokras A \& Gracia CR 2013 Response to ovarian stimulation in patients facing gonadotoxic therapy. Reproductive Biomedicine Online 26 337-344. (doi:10.1016/j.rbmo.2013.01.003)
Kohler TS, Kondapalli LA, Shah A, Chan S, Woodruff TK \& Brannigan RE 2011 Results from the survey for preservation of adolescent reproduction (SPARE) study: gender disparity in delivery of fertility preservation message to adolescents with cancer. Journal of Assisted Reproduction and Genetics 28 269-277. (doi:10.1007/s10815-010-9504-6)

Kreeger PK, Woodruff TK \& Shea LD 2003 Murine granulosa cell morphology and function are regulated by a synthetic Arg-Gly-Asp matrix. Molecular and Cellular Endocrinology 205 1-10. (doi:10.1016/ S0303-7207(03)00209-0)

Kreeger PK, Fernandes NN, Woodruff TK \& Shea LD 2005 Regulation of mouse follicle development by follicle-stimulating hormone in a threedimensional in vitro culture system is dependent on follicle stage and dose. Biology of Reproduction 73 942-950. (doi:10.1095/biolreprod. 105.042390)

Laronda MM, Duncan FE, Hornick JE, Xu M, Pahnke JE, Whelan KA, Shea LD \& Woodruff TK 2014 Alginate encapsulation supports the growth and differentiation of human primordial follicles within ovarian cortical tissue. Journal of Assisted Reproduction and Genetics 31 1013-1028. (doi:10.1007/s10815-014-0252-x)

Levine JM, Kelvin GF, Quinn GP \& Gracia CR 2015 Infertility in reproductive-age female cancer survivors. Cancer 121 1532-1539. (doi:10.1002/cncr.29181)

Lobo RA 2005 Potential options for preservation of fertility in women. New England Journal of Medicine 353 64-73. (doi:10.1056/ NEJMra043475)

Loren AW, Mangu PB, Beck LN, Brennan L, Magdalinski AJ, Partridge AH, Quinn G, Wallace WH \& Oktay K 2013 Fertility preservation for patients with cancer: American Society of Clinical Oncology clinical practice guideline update. Journal of Clinical Oncology 31 2500-2510. (doi:10.1200/JCO.2013.49.2678)

Meirow D, Levron J, Eldar-Geva T, Hardan I, Fridman E, Zalel Y, Schiff E \& Dor J 2005 Pregnancy after transplantation of cryopreserved ovarian tissue in a patient with ovarian failure after chemotherapy. New England Journal of Medicine 353 318-321. (doi:10.1056/NEJMc055237)

Murphy D, Sawczyn KK \& Quinn GP 2012 Using a patient-centered approach to develop a fertility preservation brochure for pediatric oncology patients: a pilot study. Journal of Pediatric and Adolescent Gynecology 25 114-121. (doi:10.1016/j.jpag.2011.10.007)

National Cancer Institute \& Surveillance Epidemiology and End Results Program 2014a SEER Stat Fact Sheets: All Cancer Sites (accessed 4th March 2015). http://seer.cancer.gov/statfacts/html/all.html

National Cancer Institute \& Surveillance Epidemiology and End Results Program 2014b SEER Cancer Statistics Review 1975-2011 (accessed 4th March 2015). http://seer.cancer.gov/statfacts/html/all.html

Nieman CL, Kazer R, Brannigan RE, Zoloth LS, Chase-Lansdale PL, Kinahan K, Dilley KJ, Roberts D, Shea LD \& Woodruff TK 2006 Cancer survivors and infertility: a review of a new problem and novel answers. Journal of Supportive Oncology 4 171-178.

Nieman CL, Kinahan KE, Yount SE, Rosenbloom SK, Yost KJ, Hahn EA, Volpe T, Dilley KJ, Zoloth L \& Woodruff TK 2007 Fertility preservation and adolescent cancer patients: lessons from adult survivors of childhood cancer and their parents. Cancer Treatment and Research 138 201-217. (doi:10.1007/978-0-387-72293-1_15)

Orwig KE \& Schlatt S 2005 Cryopreservation and transplantation of spermatogonia and testicular tissue for preservation of male fertility. Journal of the National Cancer Institute. Monographs 34 51-56. (doi:10. 1093/jncimonographs/gi029)

Pangas SA, Saudye H, Shea LD \& Woodruff TK 2003 Novel approach for the three-dimensional culture of granulosa cell-oocyte complexes. Tissue Engineering 9 1013-1021. (doi:10.1089/107632703322495655)

Poirot C, Abirached F, Prades M, Coussieu C, Bernaudin F \& Piver P 2012 Induction of puberty by autograft of cryopreserved ovarian tissue. Lancet 379 588. (doi:10.1016/S0140-6736(11)61781-9)

Quinn GP \& Vadaparampil ST 2013 More research, more responsibility: the expansion of duty to warn in cancer patients considering fertility preservation. American Journal of Obstetrics and Gynecology 209 98-102. (doi:10.1016/j.ajog.2013.02.031)

Quinn GP, Vadaparampil ST, Malo T, Reinecke J, Bower B, Albrecht T \& Clayman ML 2012 Oncologists' use of patient educational materials about cancer and fertility preservation. Psycho-oncology 21 1244-1249. (doi:10.1002/pon.2022) 
Redig AJ, Brannigan R, Stryker SJ, Woodruff TK \& Jeruss JS 2011 Incorporating fertility preservation into the care of young oncology patients. Cancer 117 4-10. (doi:10.1002/cncr.25398)

Reuter K, Ehmcke J, Stukenborg JB, Simoni M, Damm OS, Redmann K, Schlatt S \& Wistuba J 2014 Reassembly of somatic cells and testicular organogenesis in vitro. Tissue \& Cell 46 86-96. (doi:10.1016/j.tice.2013. 12.001)

Rodrigues JK, Navarro PA, Zelinski MB, Stouffer RL \& Xu J 2015 Direct actions of androgens on the survival, growth and secretion of steroids and anti-Müllerian hormone by individual macaque follicles during threedimensional culture. Human Reproduction 30 664-674. (doi:10.1093/ humrep/deu335)

Rodriguez S, Campo-Engelstein L, Tingen C \& Woodruff T 2011 An obscure rider obstructing science: the conflation of parthenotes with embryos in the Dickey-Wicker amendment. American Journal of Bioethics 11 20-28. (doi:10.1080/15265161.2010.546472)

Schlatt S, Ehmcke J \& Jahnukainen K 2009 Testicular stem cells for fertility preservation: preclinical studies on male germ cell transplantation and testicular grafting. Pediatric Blood \& Cancer 53 274-280. (doi:10.1002/ pbc.22002)

Scott-Trainer J 2010 The role of a patient navigator in fertility preservation. Cancer Treatment and Research 156 469-470. (doi:10.1007/978-1. 4419-6518-9)

Silva GM, Rossetto R, Chaves RN, Duarte AB, Araujo VR, Feltrin C, Bernuci MP, Anselmo-Franci JA, Xu M, Woodruff TK et al. 2014 In vitro development of secondary follicles from pre-pubertal and adult goats cultured in two-dimensional or three-dimensional systems. Zygote $\mathbf{2 3}$ 475-484. (doi:10.1017/S0967199414000070)

Sklar CA, Mertens AC, Mitby P, Whitton J, Stovall M, Kasper C, Mulder J, Green D, Nicholson HS, Yasui Y et al. 2006 Premature menopause in survivors of childhood cancer: a report from the Childhood Cancer Survivor Study. Journal of the National Cancer Institute 98 890-896. (doi:10.1093/jnci/djj243)

Skory RM, Xu Y, Shea LD \& Woodruff TK 2015 Engineering the ovarian cycle using in vitro follicle culture. Human Reproduction 30 1386-1395. (doi:10.1093/humrep/dev052)

Smeyers C, Wallach H \& Woodruff TK 2012 Repropedia: a reproductive lexicon to fill the gap in reproductive terminology. Biology of Reproduction 87 98. (doi:10.1095/biolreprod.112.104000)

Smith MA, Altekruse SF, Adamson PC, Reaman GH \& Seibel NL 2014 Declining childhood and adolescent cancer mortality. Cancer 120 2497-2506. (doi:10.1002/cncr.28748)

Songsasen N, Woodruff TK \& Wildt DE 2011 In vitro growth and steroidogenesis of dog follicles are influenced by the physical and hormonal microenvironment. Reproduction 142 113-122. (doi:10.1530/ REP-10-0442)

Tagler D, Tu T, Smith RM, Anderson NR, Tingen CM, Woodruff TK \& Shea LD 2012 Embryonic fibroblasts enable the culture of primary ovarian follicles within alginate hydrogels. Tissue Engineering. Part A $\mathbf{1 8}$ 1229-1238. (doi:10.1089/ten.tea.2011.0418)

Tingen C, Rodriguez S, Campo-Engelstein L \& Woodruff TK 2010 Research funding. Politics and parthenotes. Science 330 453. (doi:10.1126/ science.1196881)

Tingen CM, Kiesewetter SE, Jozefik J, Thomas C, Tagler D, Shea L \& Woodruff TK 2011 A macrophage and theca cell-enriched stromal cell population influences growth and survival of immature murine follicles in vitro. Reproduction 141 809-820. (doi:10.1530/REP-10-0483)

Valli H, Phillips BT, Shetty G, Byrne JA, Clark AT, Meistrich ML \& Orwig KE 2014 Germline stem cells: toward the regeneration of spermatogenesis. Fertility and Sterility 101 3-13. (doi:10.1016/j.fertnstert.2013.10.052)

Waimey KE, Duncan FE, Su HI, Smith K, Wallach H, Jona K, Coutifaris C, Gracia CR, Shea LD, Brannigan RE et al. 2013 Future directions in oncofertility and fertility preservation: a report from the 2011 Oncofertility Consortium Conference. Journal of Adolescent and Young Adult Oncology 2 25-30. (doi:10.1089/jayao.2012.0035)

Wallace WH, Smith AG, Kelsey TW, Edgar AE \& Anderson RA 2014 Fertility preservation for girls and young women with cancer: population-based validation of criteria for ovarian tissue cryopreservation. Lancet. Oncology 15 1129-1136. (doi:10.1016/S1470-2045(14)70334-1)

West ER, Xu M, Woodruff TK \& Shea LD 2007 Physical properties of alginate hydrogels and their effects on in vitro follicle development. Biomaterials 28 4439-4448. (doi:10.1016/j.biomaterials.2007.07.001)
Woodruff TK 2010 The Oncofertility Consortium - addressing fertility in young people with cancer. Nature Reviews. Clinical Oncology 7 466-475. (doi:10.1038/nrclinonc.2010.81)

Woodruff TK 2013 From the bench to bedside to babies: translational medicine made possible by funding multidisciplinary team science. Journal of Assisted Reproduction and Genetics 30 1249-1253. (doi:10. 1007/s10815-013-0082-2)

Woodruff TK \& Shea LD 2011 A new hypothesis regarding ovarian follicle development: ovarian rigidity as a regulator of selection and health. Journal of Assisted Reproduction and Genetics 28 3-6. (doi:10.1007/ s10815-010-9478-4)

Woodruff TK \& Snyder KA 2007 In Oncofertility: Fertility Preservation for Cancer Survivors. New York, NY: Springer Science+Business Media.

Woodruff TK, Zoloth L, Campo-Engelstein L \& Rodriguez S 2010 In Oncofertility: Ethical, Legal, Social, and Medical Perspectives. New York, NY: Springer Science+ Business Media.

Woodruff TK, Clayman ML \& Waimey KE 2013 In Oncofertility Communication: Sharing Information and Building Relationships Across Disciplines. New York, NY: Springer Science+Business Media.

World Health Organisation 2015 Cancer Fact Sheet (accessed 4th March 2015).

Xu M, Kreeger PK, Shea LD \& Woodruff TK 2006 Tissue-engineered follicles produce live, fertile offspring. Tissue Engineering 12 2739-2746. (doi:10. 1089/ten.2006.12.2739)

Xu M, Banc A, Woodruff TK \& Shea LD 2009a Secondary follicle growth and oocyte maturation by culture in alginate hydrogel following cryopreservation of the ovary or individual follicles. Biotechnology and Bioengineering 103 378-386. (doi:10.1002/bit.22250)

Xu M, Barrett SL, West-Farrell E, Kondapalli LA, Kiesewetter SE, Shea LD \& Woodruff TK $2009 \mathrm{~b}$ In vitro grown human ovarian follicles from cancer patients support oocyte growth. Human Reproduction 24 2531-2540. (doi:10.1093/humrep/dep228)

Xu M, West-Farrell ER, Stouffer RL, Shea LD, Woodruff TK \& Zelinski MB 2009c Encapsulated three-dimensional culture supports development of nonhuman primate secondary follicles. Biology of Reproduction 81 587-594. (doi:10.1095/biolreprod.108.074732)

Xu J, Bernuci MP, Lawson MS, Yeoman RR, Fisher TE, Zelinski MB \& Stouffer RL 2010 Survival, growth, and maturation of secondary follicles from prepubertal, young, and older adult rhesus monkeys during encapsulated three-dimensional culture: effects of gonadotropins and insulin. Reproduction 140 685-697. (doi:10.1530/REP-10-0284)

Xu M, Pavone ME \& Woodruff T 2011a Fruitful progress to fertility: preserving oocytes from chemodestruction. Nature Medicine 17 1562-1563. (doi:10.1038/nm.2595)

Xu M, Fazleabas AT, Shikanov A, Jackson E, Barrett SL, Hirshfeld-Cytron J, Kiesewetter SE, Shea LD \& Woodruff TK 2011b In vitro oocyte maturation and preantral follicle culture from the luteal-phase baboon ovary produce mature oocytes. Biology of Reproduction 84 689-697. (doi:10.1095/biolreprod.110.088674)

Xu J, Lawson MS, Yeoman RR, Pau KY, Barrett SL, Zelinski MB \& Stouffer RL 2011C Secondary follicle growth and oocyte maturation during encapsulated three-dimensional culture in rhesus monkeys: effects of gonadotrophins, oxygen and fetuin. Human Reproduction 26 1061-1072. (doi:10.1093/humrep/der049)

Xu J, Xu M, Bernuci MP, Fisher TE, Shea LD, Woodruff TK, Zelinski MB \& Stouffer RL 2013a Primate follicular development and oocyte maturation in vitro. Advances in Experimental Medicine and Biology 761 43-67.

Xu J, Lawson MS, Yeoman RR, Molskness TA, Ting AY, Stouffer RL \& Zelinski MB 2013b Fibrin promotes development and function of macaque primary follicles during encapsulated three-dimensional culture. Human Reproduction 28 2187-2200. (doi:10.1093/humrep/ det093)

Received 9 April 2015

First decision 11 May 2015

Revised manuscript received 16 June 2015

Accepted 29 June 2015 